\title{
O MODELO DE CINCO FATORES DE FAMA-FRENCH E O CRESCIMENTO ECONÔMICO FUTURO: EVIDÊNCIAS EM MERCADOS EMERGENTES
}

\author{
FAMA-FRENCH FIVE-FACTOR ASSET PRICING MODEL AND THE FUTURE \\ ECONOMIC GROWTH: EVIDENCES FROM EMERGING MARKETS
}

\author{
José Clemente Jacinto Ferreira ${ }^{1}$ \\ Universidade da Beira Interior \\ joseclemente014@gmail.com / \\ jose.clemente.ferreira@ubi.pt
}

\author{
Luiz Paulo Fávero \\ Universidade de São Paulo \\ lpfavero@usp.br
}

\author{
Ana Paula Matias Gama \\ Universidade da Beira Interior \\ amatias@ubi.pt
}

\author{
Raquel Sales da Silva Costa \\ Universidade de São Paulo \\ raquel.sales.silva@usp.br
}

\section{RESUMO}

Este estudo procura investigar se existe uma relação entre os fatores de risco do modelo de avaliação de ativos de cinco fatores proposto por Fama e French (2015) e o crescimento econômico futuro dos países emergentes que compõem o grupo denominado de BRICS, bem como o próprio grupo. Para tanto, a partir de uma amostra do Produto Interno Bruto (PIB) e dos retornos dos fatores de risco de mercado, porte, índice book-to-market, lucro operacional e investimento dos mercados de ações no período compreendido entre 1993 e 2019, aplicou-se a técnica de análise de regressão quantílica para os percentis 0,$05 ; 0,25 ; 0,50 ; 0,75 ;$ e 0,95 , bem como se estimaram cinco modelos de regressão simples e um de regressão múltipla. Para fins de comparação, modelos quantílicos para dados em painel com efeitos fixos também foram estimados. Os resultados empíricos sugerem que os retornos dos fatores de risco de mercados de ações emergentes captam informações que ajudam a prever o crescimento econômico dos BRICS e dos respectivos países.

Palavras-chave: Crescimento do PIB. BRICS. Retorno. Modelo de cinco fatores. Regressão quantílica.

\footnotetext{
1 Avenida Maria Lamas, 45, $3^{\circ}$ Esquerdo, Serra das Minas - 2635-434 - Rio de Mouro - Portugal. Departamento de Gestão e Economia - Estrada do Sineiro - Polo IV - 6200-209 - Covilhã - Portugal.
} 


\begin{abstract}
This study seeks to investigate whether there is a relationship between the risk factors of the five-factor asset pricing model proposed by Fama and French (2015) and the future economic growth of each of the emerging countries that compose the group known as BRICS, as well as the whole group itself. To this end, from a sample of GDP and the returns of market risk factors, size, book-to-market ratio, operating profit and investment of the stock markets from 1993 to 2019, we estimate quantile regression models with percentiles 0.05, 0.25, 0.50, 0.75 and 0.95, as well as five simple regression models and one multiple regression model. In order to allow one to establish comparisons, we also estimate fixed effects panel data quantile models. Empirical results suggest that the returns of stock market risk factors capture information that helps predict the economic growth of BRICS and the correspondent countries.
\end{abstract}

Keywords: GDP growth. BRICS. Return. Five-factor asset pricing model. Quantile regression.

\title{
1 INTRODUÇÃO
}

Um número considerável de estudos desenvolvidos com maior consistência a partir da década de 1980 evidencia que as variações dos retornos das ações desempenham um papel relevante na previsão do crescimento econômico. Com o desenvolvimento de duas correntes teórico-empíricas que constituem os fundamentos das finanças modernas, a teoria de avaliação de ativos que decorre da teoria da seleção da carteira formulada por Markowitz (1959) e a teoria de eficiência de mercados proposta por Fama (1970), um novo olhar sobre a relação entre o crescimento econômico e os mercados de ações foi introduzido por Liew e Vassalou (2000) à luz dos modelos de avaliação de ativos Capital Asset Pricing Model (CAPM) desenvolvido de forma independente por Sharpe (1964) e Lintner (1965) e do modelo de três fatores de risco proposto por Fama e French (1993).

Liew e Vassalou (2000) atestaram que os fatores de risco correspondentes à dimensão da empresa $(S M B)$ e ao índice book-to-market $(H M L)$ ajudaram a prever o crescimento econômico futuro de dez países desenvolvidos, independentemente de estes fatores estarem associados ao fator de risco de mercado. Hanhardt e Ansotegui Olcoz (2008) documentam, adicionalmente, que o fator de risco $S M B$ contém informação que ajuda a prever o crescimento econômico da Zona do Euro.

No entanto, apesar das evidências empíricas (Capaul, Rowley e Sharpe, 1993; Fama French, 1996, 1998) a favor do modelo de três fatores de Fama e French (1993), Fama e French (2015) apresentaram um modelo de avaliação de ativos formado por cinco fatores de risco, mercado $(M K T)$, dimensão $(S M B)$, índice book-to-market $(H M L)$, lucro operacional $(R M W) \mathrm{e}$ investimento $(C M A)$ e, a partir de estudos internacionais, Fama e French $(2012,2017)$ observaram que mercados moderadamente integrados podem oferecer os mesmos fatores de risco, porém de maneira diferenciada em cada mercado regional.

Apesar do modelo de avaliação de ativos de cinco fatores ter sido utilizado e verificado por meio de estudos aplicados a diversos mercados emergentes (Singh \& Yadav, 2015; Erdinc, 2017; Guo et al., 2017; Karaomer, 2017; Lin, 2017; Zaremba \& Czapkiewicz, 2017; Leite et al., 2018; Oskan, 2018), no contexto de integração dos mercados de ações emergentes não existe nenhum estudo empírico que analisa a relação entre os fatores de risco e o crescimento econômico futuro. Sob a hipótese de integração regional dos mercados de ações, Ferreira e Gama (2020) atestaram a existência de relação positiva entre os fatores de risco do modelo de avaliação de ativos de Fama e French $(2015,2017)$ e o crescimento econômico futuro de países com mercados de ações desenvolvidos.

Moer (2005) observou que, em mercados de ações de grande dimensão, os fatores de risco regionais apresentam melhor desempenho para o cálculo do retorno esperado quando 
comparado a fatores de risco domésticos. Brooks e Del Negro (2005) constataram que metade da variação dos retornos produzida pelos mercados domésticos desenvolvidos e emergentes é devida a eventos de natureza regional, e a redução do risco pode ser obtida por meio de carteiras diversificadas de ações regionais.

Assim sendo, o objetivo deste estudo consiste em analisar, por meio da técnica de regressão quantílica para os percentis 0,$05 ; 0,25 ; 0,50 ; 0,75$; e 0,95 , se os fatores de risco de mercado de ações emergentes do modelo de avaliação de ativos proposto por Fama e French (2015) captam informações que ajudam a prever o crescimento econômico futuro, representado pelo Produto Interno Bruto (PIB) dos cinco países que compõem os BRICS, ou seja, Brasil, Rússia, Índia, China e África do Sul, assim como o PIB conjunto destes países. A escolha desta amostra inclui países de diferentes continentes e sub-regiões que, no conjunto de países de mercados de ações emergentes, conforme a classificação do Morgan Stanley Capital International (MSCI), apresentam alto potencial de desenvolvimento econômico. A motivação para o uso da técnica de regressão quantílica resulta da natureza da distribuição assimétrica da taxa de crescimento do PIB, caracterizada por recorrentes flutuações (em torno da sua linha de tendência de crescimento de longo prazo) que refletem períodos de ciclos econômicos adversos cujos diferentes comportamentos que ocorrem nos percentis da distribuição condicional não são observáveis no contexto de modelos de regressão à média como, por exemplo, aqueles estimados por mínimos quadrados ordinários. Assim sendo, por meio da regressão quantílica procura-se analisar o comportamento da taxa de crescimento do PIB, condicional aos retornos dos fatores de risco de mercados de ações emergentes.

Os resultados obtidos indicam que os modelos de avaliação de ativos desenvolvidos para estimar o custo de capital e carteiras de investimento podem servir de indicadores econômicos, pois fornecem informações que ajudam a prever o crescimento econômico. Estas evidências estão em linha com as observações de Fama e French (2015) que argumentam que, embora os fatores que resultam dos retornos das ações dos modelos de avaliação de ativos cinco fatores de risco não serem em si variáveis de estado, os retornos médios esperados de carteiras diversificadas refletem de fato as variáveis de estado não identificadas que descrevem um conjunto de oportunidades de investimento, no contexto de um modelo multifatorial como o Intertemporal Capital Asset Pricing Model (ICAPM), proposto por Merton (1973).

O presente estudo está estruturado em seis seções. Segue-se a seção dois, designada de referencial teórico, em que se procura expor os aspetos teóricos empíricos produzidos nos últimos anos. Na seção três apresentam-se os critérios e os métodos de análise para o presente estudo. Na seção quatro apresenta-se a descrição dos dados. Seguem-se análise e discussão dos resultados e, por fim, a conclusão.

\section{REFERENCIAL TEÓRICO}

A teoria do ciclo econômico, que descreve a variação da atividade econômica classifica o preço das ações como um indicador que antecipa as flutuações recorrentes do desempenho econômico futuro (Schwert, 1989; Fama, 1990; Chauvet, 1999). Assim, com uma estrutura de defasagem para períodos de curto ou longo prazo (Fama, 1990; Morck et al.,1990, Zarnowitz, 1992), a previsão do crescimento econômico pode ser feita por meio do retorno do mercado de ações. De fato, a literatura econômico-financeira atesta que a previsão do crescimento econômico pode ser feita por meio do retorno do mercado de ações (Fama, 1981, 1990; Fischer \& Merton, 1984; Kaul, 1987; Schwert, 1990; Aylward \& Glen, 2000; Mauro; 2003; Panopoulou, 2009) e pelos fatores de risco dos modelos de avaliação de ativos (Chen, Roll \& Ross 1986; Liew \& Vassalou, 2000; Neves e Leal, 2003; Hanhardt \& Ansotegui Olcoz, 2008). 
No contexto da taxa de retorno do mercado de ações, diversos estudos atestaram a existência de uma relação positiva entre retorno das ações e crescimento econômico futuro. Fama $(1981,1990)$ documentou a relação positiva e estatisticamente significante entre a variação do retorno anual e o crescimento econômico americano. Kaul (1987) atestou uma relação positiva entre o retorno das ações e o crescimento econômico futuro para os mercados americano (1953 a 1983), canadense (1952 a 1983), alemão e britânico (1958 a 1983). Schwert (1990) estendeu a análise para o período de 1889 a 1988 e confirmou as evidências de Fama (1981, 1990) para o mercado americano. Aylward e Glen (2000) relataram a capacidade do mercado de ações em prever o crescimento econômico de países desenvolvidos e em vias de desenvolvimento. Mauro (2003) observou uma relação positiva entre o crescimento do PIB e o retorno do mercado de ações de um conjunto de países de economias desenvolvida e emergente para o período de 1977 a 1998. Panopoulou (2009) atestou a importância do mercado de ações para a previsão do crescimento econômico de doze países da Zona do Euro $^{2}$ para o período de janeiro de 1988 a maio de 2005.

À luz dos modelos de avaliação de ativos merece destaque o estudo de Liew e Vassalou (2000). Os autores constataram que, tal como o fator de mercado, os fatores de risco $S M B$ e $H M L$, independentemente de estarem associados ao risco de mercado, encerram informações que ajudam a prever o crescimento econômico futuro de dez países de mercados de ações desenvolvidos entre 1978 e 1996. Estes resultados foram confirmados por Neves e Leal (2003), que atestaram uma relação positiva entre os fatores de risco $S M B$ e $H M L$ e o crescimento econômico futuro do Brasil para o período de 1986 a 2001. Hanhardt e Ansotegui Olcoz (2008) utilizaram os fatores de risco $M K T$, SMB, HML e WML (momento) de carteiras de ações de cinco tipos de indústrias de doze países da Zona do Euro para o período de 1995 a 2003, documentando que o fator de risco de efeito $S M B$ contém informação robusta que ajuda a prever o crescimento econômico da Zona do Euro. Liew e Vassalou (2000) investigaram a relação entre o PIB e os fatores MKT, SMB e HML nos Estados Unidos para o período de 1957 a 1998. Os autores constataram, por meio da esimação de modelos de regressão linear simples, que os fatores $M K T$ e $S M B$ apresentam coeficientes positivos, porém a significância estatística ao nível de $1 \%$ foi verificada apenas para o fator $M K T$. Já por meio da estimação de modelos de regressão múltipla, os fatores de risco $M K T, S M B$ e $H M L$ apresentaram parâmetros positivos, com significância estatística ao nível de $10 \%$ para a varável $M K T$. Adicionalmente, Liu e Di Iorio (2013) observaram que os fatores de risco $S M B$ e $H M L$ antecipam o crescimento futuro australiano para o período de 1993 a 2010.

Fama e French (2015) ampliaram o modelo de avaliação de ativos de três para cinco fatores de risco, adicionando os fatores referentes ao lucro operacional $(R M W)$ resultante da diferença entre retornos de carteiras diversificadas de ações de empresas com elevado lucro operacional em relação às empresas com reduzido lucro operacional, e ao fator investimento $(C M A)$ resultante da diferença entre retornos de carteiras diversificadas de ações de empresas com baixo e alto investimento, tal como apresentado na equação (Eq. 1).

$$
R_{i t}-R_{f t}=\alpha_{i}+\beta_{i}\left(R_{m t}-R_{f t}\right)+\beta_{i s} S M B_{t}+\beta_{i h} H M L_{t}+\beta_{i r} R M W_{t}+\beta_{i c} C M A_{t}+\varepsilon_{i t}
$$

em que $R_{i t}-R_{f t}$ representa o retorno em excesso do ativo ou carteira $i$ no instante de tempo $t ; \beta_{i}$ representa o risco de mercado; $R_{f t}$ é a taxa livre de risco no instante de tempo $t ;\left(R_{m t}-R_{f t}\right)$ é o prêmio de risco que corresponde ao retorno médio em excesso da carteira de mercado $S M B_{t}$ (Small minus Big) que representa a diferença entre retornos de carteiras diversificadas de ações de empresas pequenas em relação às grandes; $H M L_{t}$ (High minus Low) é a diferença entre

\footnotetext{
${ }^{2}$ Alemanha, Áustria, Bélgica, Espanha, Finlândia, França, Grécia, Holanda, Irlanda, Itália, Luxemburgo e Portugal.
} 
retornos de carteiras diversificadas de ações da razão book-to-market alta e baixa; $R M W_{t}$ (Robust Minus Weak) é o fator lucro operacional que resulta da diferença entre retornos de carteiras diversificadas de ações de empresas com elevado lucro operacional rem relação às empresas com reduzido lucro operacional; fator investimento $C M A_{t}$ (Conservative Minus Aggressive) resulta da diferença entre retornos de carteiras diversificadas de ações de empresas de baixo e alto investimento; e $\varepsilon_{i t}$ representa os termos de erro.

Por meio de estudos internacionais, Fama e French $(2012,2017)$ observaram que mercados moderadamente integrados podem deter os mesmos fatores de risco. Contudo, dependendo da série histórica, os fatores de risco atuam de maneira diferente em cada mercado. Ferreira e Gama (2020), sob a hipótese de integração regional dos mercados de ações desenvolvidos e utilizando dados de janeiro 1991 a dezembro de 2018, encontraram evidências de que os fatores de risco $M K T, S M B, H M L, R M W$ e $C M A$ ajudam a prever o crescimento econômico futuro das regiões da América do Norte, Ásia Pacífico e Europa, assim como o crescimento econômico doméstico da Alemanha, Canadá, Estados Unidos, França, Hong Kong e Singapura.

\section{MÉTODO}

O objetivo desta pesquisa consiste em analisar se os fatores de risco de mercados de ações emergentes do modelo de avaliação de ativos de cinco fatores formulado por Fama e French (2015) ajudam a prever o crescimento econômico conjunto dos BRICS e de cada um dos cinco países que compõe o grupo, Brasil, Rússia, Índia, China e África do Sul, ou seja, procura-se investigar a existência de uma relação positiva entre o crescimento econômico e os cinco fatores de risco do modelo de avaliação de ativos de Fama e French (2015). A escolha desta amostra inclui países de diferentes continentes e sub-regiões que, no conjunto de países de mercados de ações emergentes, conforme a classificação do $M S C I$, apresentam alto potencial de desenvolvimento econômico. Conforme discutido, os estudos empíricos aplicados a estes mercados têm identificado fatores de risco do modelo de avaliação de ativos de cinco fatores.

Para o efeito, utiliza-se a técnica de regressão quantílica para analisar a relação entre a distribuição assimétrica da taxa de crescimento do PIB condicional aos fatores de risco nos percentis, 0,$05 ; 0,25 ; 0,50 ; 0,75$; e 0,95 . Os percentis 0,05 e 0,25 representam as taxas de crescimento mais baixas, o percentil 0,50 denota taxa de crescimento mediana; e os percentis 0,75 e 0,95 representam as taxas de crescimento mais altas. Para efeito de comparação da magnitude e sinal dos coeficientes, será estimado também o modelo de regressão pelo método de mínimos quadrados ordinários (MQO).

Como especificado, a motivação para a utilização da técnica de regressão quantílica parte da natureza da distribuição assimétrica da variável dependente, PIB, na qual permite analisar os efeitos dos fatores de risco nos diferentes percentis condicionais.

A regressão quantílica, proposta por Koenker e Bassett (1978) caracteriza-se pela minimização da soma ponderada dos resíduos absolutos e estima os parâmetros do modelo de regressão nos diferentes percentis (entre 0 e 1 ) da variável dependente, condicional ao vetor de variáveis preditoras. A mediana descreve a melhor medida de tendência central, visto que não é afetada pelos valores extremos e outliers, ao contrário da média, medida considerada na estimação de modelos de regressão por mínimos quadrados ordinários (Fávero e Belfiore, 2017). Assim sendo, conforme Koenker e Bassett (1978) e Buchinsky, (1998) a regressão quantílica apresenta-se como (i) técnica de regressão robusta a outliers, cujos estimadores dos parâmetros não são influenciados por dados extremos, (ii) não há a necessidade de se verificar o pressuposto da normalidade dos resíduos, e (iii) fornece informação mais completa que 
permite explicar e prever o comportamento da variável dependente, dadas as variações ocorridas nas variáveis preditoras.

Liew e Vassalou (2000), por meio da técnica de regressão por MQO, observaram que os fatores de risco domésticos $M K T, S M B$ e $H M L$, de forma independente, ajudam a prever o crescimento econômico de países desenvolvidos. Ferreira e Gama (2020), sob a hipótese de integração regional dos mercados de ações desenvolvidos e fazendo uso da técnica de regressão quantílica, atestaram relação positiva entre os fatores de risco do modelo de avaliação de ativos de cinco fatores proposto por Fama e French $(2015,2017)$ e o crescimento econômico futuro de países de mercados de ações desenvolvidos. No presente estudo, estende-se a análise para cada um dos cinco países de mercados de ações emergentes dos BRICS.

Seguindo Liew e Vassalou (2000) e Ferreira e Gama (2020), a análise será feita em duas etapas. A primeira consiste na estimação de cinco modelos de regressão simples, associado a cada um dos fatores de risco, com o objetivo de verificar se os retornos do período $t-1$ de cada fator de risco, individualmente, capta informações sobre o crescimento econômico futuro anual para o conjunto dos BRICS, representado pela equação (Eq. 2).

$$
\text { PIB }_{t}=\alpha+\beta \text { Fator }_{t-1}+\varepsilon_{t}
$$

em que, $P I B_{t}$ denota a taxa de crescimento do Produto Interno Bruto para o período $t$, calculada de forma logarítmica; Fator $_{t-1}$, representa os retornos dos fatores de risco $M K T, S M B, H M L$, $R M W$ e $C M A$ no período anterior, e $\varepsilon_{t}$ representa os termos de erro do modelo de regressão.

Zarnowitz (1992) argumentou que os preços das ações tendem a apresentar fortes tendências cíclicas porque atuam como indicadores que antecipam ou refletem os movimentos iniciais das rentabilidades e inovações, como a inversão da curva de juros. Fama $(1981 ; 1990)$ justificou o impacto das variáveis macroeconômicas sobre o retorno das ações argumentando que a relação entre atividade econômica e a variação dos retornos das ações ocorre com maior consistência em um horizonte temporal de um ano (no presente estudo utiliza-se uma defasagem de um ano). A segunda etapa consiste na estimação de um modelo de regressão múltipla representado pela equação (Eq. 3), que inclui os cinco fatores de risco, para o conjunto dos BRICS e para cada um dos cinco países individualmente.

$$
P I B_{t}=\alpha+\beta_{1} M K T_{t-1}+\beta_{2} S M B_{t-1}+\beta_{3} H M L_{t-1}+\beta_{4} R M W_{t-1}+\beta_{5} C M A_{t-1}+\varepsilon_{t}
$$

O fator risco $M K T$ é formado pela diferença entre o retorno do índice de capitalização na respectiva bolsa e o título do tesouro americano mensal (CAPM). O fator de risco SMB corresponde à capitalização de cada empresa na respectiva bolsa, e resulta do produto entre preço da ação e o número de ações em circulação. O Fator de risco $H M L$ resulta da divisão entre o valor contábil da empresa e o valor da capitalização da empresa em sua respectiva bolsa. $\mathrm{O}$ fator de risco $R M W$ resulta da divisão entre o lucro operacional (receita menos custo das mercadorias vendidas, despesas com juros e vendas, despesas gerais e despesas administrativas) do período $t$ e o valor do patrimônio líquido da empresa referente ao período $t$. $\mathrm{O}$ fator de risco $C M A$ é calculado a partir da divisão da diferença do valor total de ativos do período $t-1$ e $t-2$ pelo total de ativos do período $t-2$.

Os fatores de risco $S M B, H M L, R M W$ e $C M A$ são construídos no final do mês de junho de cada ano, com base em seis carteiras ponderadas formadas pela interseção de dois grupos $S M B$ e três grupos $H M L, R M W$ e $C M A$. Os dois grupos $S M B$ são separados pelos percentis 0,90 e 0,10 . O percentil 0,90 representa o grupo de ações de grande dimensão e o percentil 0,10 representa o grupo de ações classificadas como sendo de pequena dimensão. Os três grupos para cada fator de risco $H M L, R M W$ e $C M A$, são separados pelos percentis 0,30 e 0,70 . Desta forma, conforme o fator de risco, o grupo de ações com valores abaixo do percentil 0,30 será 
classificado como sendo baixo/fraco/conservador (low weak e conservative), entre os percentis 0,30 e 0,70 será classificado como neutro $(\mathrm{N})$, e acima do percentil 0,70 , como alto/robusto/agressivo (high, robust e aggressive). Assim, esta divisão permitirá formar seis carteiras, 2x3 (Small, S e Big, B; Low, L, Neutral, N e High, H) para cada grupo (SMB e HML, $S M B$ e $R M W$, e $S M B$ e $C M A$ ), por meio da interseção de dois grupos de ativos (Small, $\mathrm{S}$ e Big, B) formados por dimensão da empresa, e três grupos de ações (Low, L, Neutral, N e High, H) formados por book-to-market, lucro operacional e investimento.

Assim, $S M B$ é formado pela diferença entre os retornos médios de nove carteiras de ações de pequenas dimensões e nove carteiras de ações de grandes dimensões de SMB book-tomarket (B/M), $S M B$ lucro operacional (OP) e $S M B$ investimento (INV), representado pelas equações (Eq. 4 a 7$)$.

$$
\begin{aligned}
& S M B_{B / M}=1 / 3(S L+S N+S H)-1 / 3(B L+B N+B H) \\
& S M B_{O P}=1 / 3(S W+S N+S R)-1 / 3(B W+B N+B R) \\
& S M B_{I N V}=1 / 3(S C+S N+S A)-1 / 3(B C+B N+B A) \\
& S M B=1 / 3\left(S M B_{B / M}+S M B_{O P}+S M B_{I N V}\right)
\end{aligned}
$$

Os fatores de risco $H M L, R M W$ e $C M A$ são formados pela diferença entre a média dos retornos de duas carteiras de ações de alto $(\mathrm{H})$ e baixo $(\mathrm{L})$ book-to-market, lucro operacional robusto $(\mathrm{R})$ e fraco $(\mathrm{W})$ e investimento conservador $(\mathrm{C})$ e agressivo (A), representados pelas equações (Eq. 8 a 10).

$$
\begin{aligned}
& H M L=1 / 2(S H+B H)-1 / 2(S L+B L) \\
& R M W=1 / 2(S R+B R)-1 / 2(S W+B L) \\
& C M A=1 / 2(S A+B A)-1 / 2(S C+B C)
\end{aligned}
$$

\section{DESCRIÇÃO DOS DADOS}

\subsection{Amostra}

Para o presente estudo foram consideradas séries históricas de dados anuais valorizados em dólar americano para o período compreendido entre janeiro de 1993 e dezembro de 2019 referentes ao Produto Interno Bruto (PIB), a preços constantes e ano base 2010, de África do Sul, Brasil, Índia, China e Rússia, bem como os retornos dos fatores de risco de mercados de ações emergentes do qual fazem parte as ções de África do Sul, Brasil, Índia, China e Rússia. O PIB conjunto dos BRICS corresponde a soma do PIB individual dos cincos países. Os dados dos retornos dos fatores de risco e do PIB foram obtidos a partir das bases de dados de Kenneth French e do Banco Mundial.

\subsection{Estatísticas descritivas}

A Tabela 1 apresenta o resumo das estatísticas descritivas para as taxas de retorno dos fatores de risco do mercado de ações emergentes, as taxas de crescimento do PIB conjunto dos BRICS e dos respectivos países, bem como os resultados dos testes de aderência à normalidade 
(Shapiro Wilk) e estacionariedade, Augmented Dickey-Fuller (ADF) com defasagem máxima de oito lags pelo critério de informação Bayesiano de Schwarz (BIC).

Tabela 1. Resumo das estatísticas descritivas para as taxas anuais de crescimento do PIB e dos retornos dos fatores de risco de mercado de ações emergente

\begin{tabular}{|l|c|c|c|c|c|c|c|c|c|c|}
\hline \multicolumn{2}{|c|}{ Variável } & $\overline{\mathbf{X}}$ & Md & Mín & Máx & Dp & As & K & SW & ADF \\
\hline \multirow{5}{*}{} & & $\mathbf{\%}$ & $\mathbf{\%}$ & $\mathbf{\%}$ & $\mathbf{\%}$ & $\mathbf{\%}$ & & & P value & Teste t \\
\cline { 2 - 11 } & MKT & 10,715 & 4,120 & $-55,360$ & 86,370 & 34,410 & 0,454 & $-0,189$ & 0,60 & $-4,32 * * *$ \\
\cline { 2 - 11 } & SMB & 2,361 & 0,650 & $-8,820$ & 44,850 & 10,596 & 2,534 & 7,711 & 0,00 & $-8,17^{* * *}$ \\
\cline { 2 - 11 } & HML & 9,668 & 2,870 & $-10,720$ & 50,870 & 14,750 & 1,065 & 0,597 & 0,01 & $-5,13^{* * *}$ \\
\cline { 2 - 11 } & RMW & 1,184 & 2,360 & $-51,730$ & 11,990 & 12,000 & $-3,262$ & 12,229 & 0,00 & $-9,89^{* * *}$ \\
\cline { 2 - 10 } & CMA & 4,030 & 4,270 & $-20,010$ & 30,940 & 8,692 & 0,246 & 3,301 & 0,02 & $-5,58^{* * *}$ \\
\hline BRICS & PIB & 5,646 & 5,231 & 2,815 & 9,844 & 1,741 & 0,556 & $-0,113$ & 0,34 & $-2,73^{*}$ \\
\hline África do Sul & PIB & 2,551 & 2,664 & $-1,550$ & 5,452 & 1,689 & $-0,287$ & $-0,208$ & 0,81 & $-2,84^{*}$ \\
\hline Brasil & PIB & 2,479 & 2,961 & $-3,610$ & 7,258 & 2,577 & $-0,531$ & 0,187 & 0,38 & $-3,41^{* *}$ \\
\hline China & PIB & 8,937 & 8,835 & 5,931 & 13,305 & 2,026 & 0,631 & $-0,440$ & 0,13 & $-1,89$ \\
\hline Índia & PIB & 6,269 & 6,807 & 3,040 & 8,476 & 1,612 & $-0,531$ & $-1,077$ & 0,02 & $-4,56^{* *}$ \\
\hline Rússia & PIB & 1,584 & 2,505 & $-13,433$ & 9,531 & 5,760 & $-0,932$ & 0,185 & 0,04 & $-3,06 * *$ \\
\hline
\end{tabular}

Nota: $\bar{X}$, média; Md, mediana; Mìn, mínimo; Máx; máximo; Dp, desvio-padrão; As, assimetria; K, curtose; SW, Shapiro Wilk; ADF, teste Aumentado de Dickey-Fuller; ***, **, *, P < 1\%, 5\% e $10 \%$.

Fonte: Autores, 2021.

Os resultados dos testes ADF apresentados na Tabela 1 indicam ausência de raiz unitária com significância estatística ao nível de $10 \%$ para todas as séries de dados, exceto para a China. Observa-se, por meio da mesma tabela, que, para o período em análise, existem grandes diferenças entre $M K T$ e todos os fatores de risco, exceto $H M L$. O fator de risco $M K T$ apresentou uma média aritmética do retorno de $10,715 \%$. Este resultado se compara a uma média aritmética de $9,668 \%(H M L), 4,030 \%$ (CMA), 2,361\% (SMB) e 1,184\% (RMW). As medianas evidenciam que metade dos retornos do fator de risco $M K T$ encontra-se abaixo de 4,12\%, comparando a $0,65 \%$ (SMB), 2,36\% (RMW), 2,87\% (HML) e 4,27\% (CMA).

A amplitude do retorno variou entre 50,95\% (CMA) e 141,73\% (MKT). O desvio-padrão dos retornos variou entre $8,692 \%(C M A)$ e $34,41 \%(M K T)$. Os retornos dos fatores de risco apresentaram distribuição de frequência diferente de um padrão normal. A assimetria negativa verificou-se no retorno do fator de risco $R M W$. A medida de curtose foi positiva para todos os fatores de risco, exceto para $M K T$. As estatísticas do teste de normalidade indicam que apenas o fator de risco $M K T$ segue uma distribuição aderente à normalidade.

Em relação à taxa de crescimento do PIB, os resultados indicam que a média anual da taxa de crescimento do PIB conjunto dos BRICS foi de 5,646\% e, para o mesmo período em análise, a média da taxa de crescimento anual do PIB para cada país variou entre 1,584\% (Rússia) e 8,937\% (China). As medianas evidenciam que metade das taxas de crescimento do PIB conjunto dos BRICS encontra-se abaixo de 5,231\% e, para cada país, a variação foi da ordem de $2,505 \%$ (Rússia) a 8,835\% (China). O desvio-padrão para as séries de dados da taxa de crescimento logarítmica do PIB manteve-se abaixo de 6\%. A medida de assimetria indica ligeiro grau de desvio em torno da média, à esquerda e com valores de -0,287 (África do Sul), -0,531 (Brasil e Índia) e -0,932 (Rússia), e à direita com valores de 0,556 (PIB conjunto dos BRICS) e 0,631 (China). A medida de curtose foi positiva para Brasil $(0,187)$ e Rússia $(0,185)$. As estatísticas do teste de normalidade indicam que as taxas de crescimento do PIB da Índia e Rússia não seguem uma distribuição aderente à normalidade. 


\section{RESULTADOS EMPÍRICOS}

Nesta seção é analisada a relação entre os fatores de risco de mercado de ações emergentes e as taxas anuais do crescimento econômico conjunto dos BRICS e de cada um dos países, por meio da estimação de modelos de regressão quantílica para os percentis 0,$05 ; 0,25$; 0,50; 0,75; e 0,95 e de um modelo de regressão por mínimos quadrados ordinários (MQO), para fins de comparação das magnitudes e dos sinais dos parâmetros.

Para os modelos estimados por MQO que apresentaram autocorrelação e heterocedasticidade dos resíduos aplicaram-se os estimadores robustos de Newey e West (1987) e White (1980) que, embora não corrijam o erro-padrão, ajustam as bandas de significância para a estimação, eventualmente, de modelos mais parcimoniosos. Os resultados aqui reportados foram obtidos por meio da estimação dos modelos nos softwares Gretl e Stata e, por meio dos quais, verificou-se a ausência de multicolinearidade entre os fatores de risco.

A Tabela 2 apresenta os resultados de cinco modelos de regressão simples representados pela equação (Eq. 2) para desempenho econômico conjunto dos BRICS. A Tabela 3 apresenta os resultados do modelo de regressão múltipla (associado à equação (Eq. 3)), com inclusão dos cinco fatores de risco para o desempenho econômico conjunto dos BRICS e para cada um dos cinco países (África do Sul, Brasil, China, Índia e Rússia).

Tabela 2. Estimações da regressão múltipla para o modelo $P I B_{t}=\alpha+\beta_{1}$ Fator $_{t-1}$ em que Fator denota $M K T, S M B, H M L, R M W, C M A$

\begin{tabular}{|c|c|c|c|c|c|c|c|}
\hline \multirow{2}{*}{\multicolumn{2}{|c|}{ Modelo }} & \multirow[b]{2}{*}{ MQO } & \multicolumn{5}{|c|}{ Regressão quantílica } \\
\hline & & & 0,05 & 0,25 & $\mathbf{0 , 5 0}$ & 0,75 & 0,95 \\
\hline \multicolumn{8}{|c|}{ BRICS } \\
\hline 1 & MKT & $\begin{array}{l}0,022^{*} \\
(0,012)\end{array}$ & $\begin{array}{c}0,002 \\
(0,014)\end{array}$ & $\begin{array}{c}0,017 * * \\
(0,007)\end{array}$ & $\begin{array}{c}0,020 * * * \\
(0,007)\end{array}$ & $\begin{array}{c}0,029 * * * \\
(0,005)\end{array}$ & $\begin{array}{c}0,051 * * * \\
(0,004)\end{array}$ \\
\hline 2 & SMB & $\begin{array}{r}-0,023 \\
(0,033) \\
\end{array}$ & $\begin{array}{c}-0,024 \\
(0,018)\end{array}$ & $\begin{array}{l}-0,040 \\
(0,026)\end{array}$ & $\begin{array}{l}-0,037 \\
(0,022)\end{array}$ & $\begin{array}{c}0,024 \\
(0,058) \\
\end{array}$ & $\begin{array}{l}-0,030 \\
(0,073) \\
\end{array}$ \\
\hline 3 & HML & $\begin{array}{c}0,014 \\
(0,029) \\
\end{array}$ & $\begin{array}{c}-0,044 * * * \\
(0,005) \\
\end{array}$ & $\begin{array}{c}-0,030 * * \\
(0,015)\end{array}$ & $\begin{array}{c}0,047 * * \\
(0,018)\end{array}$ & $\begin{array}{c}0,082 * * * \\
(0,023)\end{array}$ & $\begin{array}{c}0,182 * * * \\
(0,023)\end{array}$ \\
\hline 4 & RMW & $\begin{array}{c}0,009 \\
(0,033)\end{array}$ & $\begin{array}{c}0,100 * * * \\
(0,022)\end{array}$ & $\begin{array}{c}0,030 * * \\
(0,013)\end{array}$ & $\begin{array}{c}0,042 * * \\
(0,017)\end{array}$ & $\begin{array}{c}-0,164 * * * \\
(0,026)\end{array}$ & $\begin{array}{c}-0,217 * * * \\
(0,009)\end{array}$ \\
\hline 5 & CMA & $\begin{array}{l}-0,026 \\
(0,040)\end{array}$ & $\begin{array}{c}0,026 * \\
(0,015)\end{array}$ & $\begin{array}{l}-0,046 \\
(0,030)\end{array}$ & $\begin{array}{c}0,012 \\
(0,031)\end{array}$ & $\begin{array}{c}-0,014 \\
(0,107)\end{array}$ & $\begin{array}{c}-0,070^{*} \\
(0,039)\end{array}$ \\
\hline
\end{tabular}

Nota: MQO, mínimos quadrados ordinários; ***, **, *, P < 1\%, 5\% e 10\%. Erros-padrão entre parênteses. Fonte: Autores, 2021.

Por meio da Tabela 2 observa-se que as estimações obtidas pela regressão quantílica indicam que, a exceção da Índia, o fator de risco $M K T$ apresentou uma relação positiva e estatisticamente significante, ao nível de $5 \%$, em quatro dos cinco percentis em análise. A assimetria da taxa de crescimento do PIB perante o fator de risco mercado $(M K T)$ variou entre $1,7 \%$ (percentil 0,25$)$ e $5,1 \%$ (percentil 0,95 ), em comparação ao valor médio $(2,2 \%$ ) obtido por meio da estimação por $\mathrm{MQO}^{3}$.

Estes resultados estão em linha com os obtidos por Ferreira e Gama (2020), que estudaram a relação entre $M K T$ e o crescimento econômico regional de países de mercados de ações desenvolvidos das regiões da América do Norte (Canadá e Estados Unidos), Ásia Pacifico

\footnotetext{
${ }^{3}$ No Apêndice são apresentados os resultados oriundos das estimações de modelos de regressão quantílica levando-se em consideração a longitudinalidade dos dados, ou seja, a estrutura em painel do dataset (Tabela 4). Neste caso, foram estimados modelos com efeitos fixos, para os mesmos percentis utilizados para a consolidação da Tabela 2, para fins de comparações. O gráfico da Figura 2, também no Apêndice, permite que se elabore a comparação visual entre as duas estimações, sem relevantes diferenças entre elas, para o percentil de $50 \%$.
} 
(Austrália, Hong Kong, Nova Zelândia e Singapura) e Europa (Alemanha, Áustria, Bélgica, Dinamarca, Espanha, Finlândia, França, Grécia, Holanda, Irlanda, Itália, Noruega, Portugal, Reino Unido, Suécia e Suíça).

As estimações obtidas por MQO para os modelos (3 e 4) univariados, tendo como variáveis preditoras os fatores de risco $H M L$ e $R M W$, evidenciam uma relação positiva com a taxa de crescimento do PIB conjunto dos BRICS, porém sem significância estatística ao nível de $10 \%$.

Em relação ao modelo 2, o fator de risco $S M B$, contrariando a estimação relizada por MQO, apresentou coeficiente positivo no percentil 0,75 , porém sem significância estatística ao nível de 10\%. Estes resultados contrariam os de Ferreira e Gama (2020), que observaram uma relação positiva de significância estatística entre um a três percentis para as regiões da América do Norte, Ásia Pacífico e Europa.

Para o modelo 3, o fator de risco $H M L$ apresentou relação positiva e estatisticamente significante, ao nível de 5\%, nos percentis mediano (percentil 0,5) e nos mais altos (percentis $0,75$ e 0,95$)$. Um pormenor importante assinala a evolução positiva dos coeficientes estimados, dos percentis mais baixos aos mais altos, ou seja, a magnitude dos coeficientes variou entre 4,4\% (percentil 0,05), 4,7\% (percentil 0,50), 8,2\% (percentil 0,75) e 18,2\% (percentil 0,95), em comparação ao valor médio de 1,4\% (sem significância estatística ao nível de 10\%) obtido por meio da estimação por MQO.

A Figura 1 ilustra o desempenho do fator de risco regional $H M L$ sobre a distribuição condicional quantílica, bem como a obtida por meio da estimação por MQO, da taxa de crescimento do PIB conjunto dos BRICS. As linhas vertical e horizontal apresentam respectivamente, os coeficientes de $H M L$ e os percentis (tau) de 0 a 1 . A linha azul contínua representa o coeficiente médio estimado obtido por meio de MQO, e as linhas azuis pontilhadas os respectivos intervalos de confiança a 95\%. A área sombreada representa os intervalos de confiança dos parâmetros obtidos por meio da estimação dos modelos de regressão quantílica, sendo a linha preta a estimação média dos parâmetros para cada um dos cinco percentis em análise.

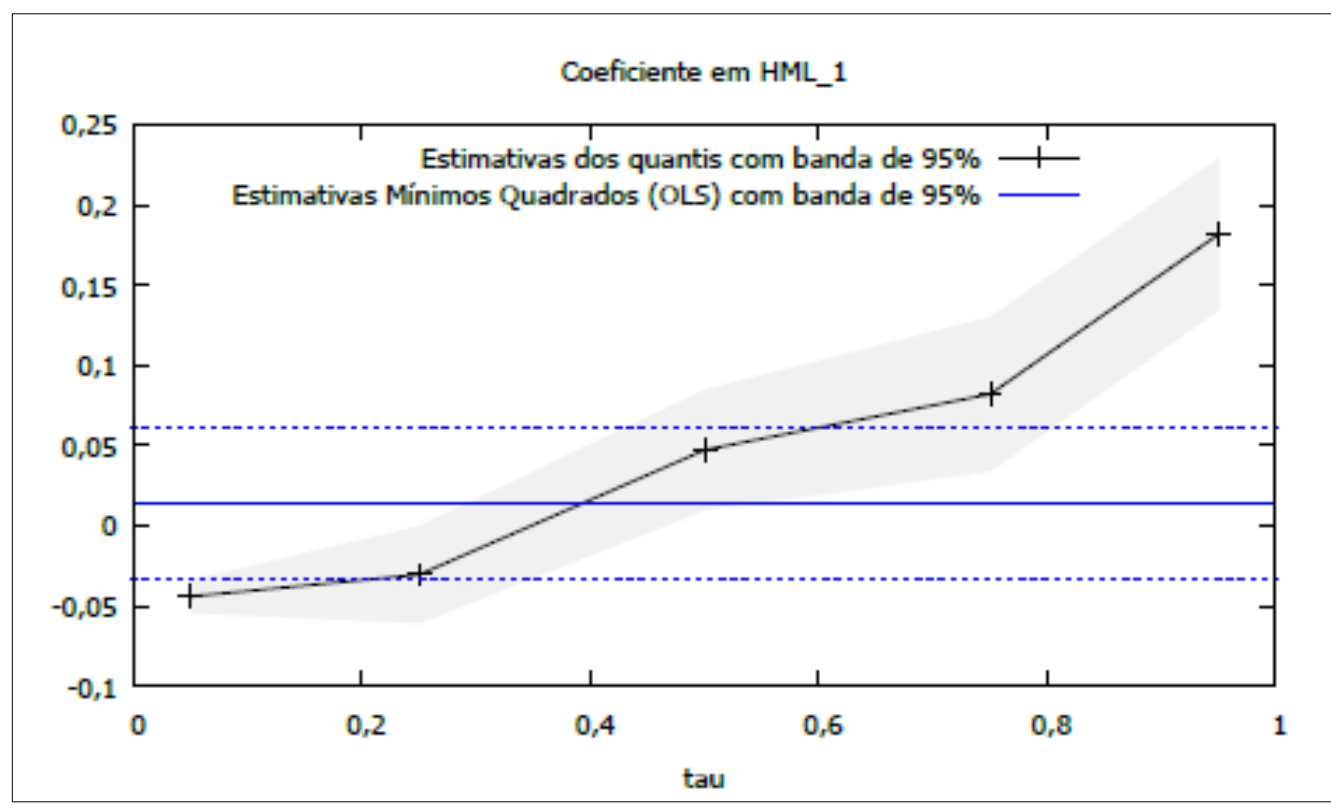

Figura 1. Desempenho do fator de risco HML sobre os percentis e média condicional do PIB conjunto dos BRICS

Fonte: Autores, 2021. 
Com efeito, o comportamento do fator de risco $H M L$ ilustrado na Figura 1 comprova os resultados apresentados na Tabela 2, por meio da qual é possível observar o desempenho crescente de $H M L$. Ferreira e Gama (2020) observaram relação positiva de significância estatística entre $H M L$ e o crescimento econômico futuro das regiões da América do Norte e Europa.

Em relação ao modelo 4, o fator de risco $R M W$ apresentou coeficientes positivos e significância estatística, ao nível de 5\%, em três dos cinco percentis em análise, com magnitude da ordem de $3 \%$ (percentil 0,25 ) a $10 \%$ (percentil 0,05 ), em comparação ao valor médio de $0,9 \%$ (sem significância estatística ao nível de $10 \%$ ) obtido por meio da estimação por MQO. Ferreira e Gama (2020) observaram relação positiva e significância estatística, ao nível de 5\%, entre $R M W$ e o crescimento econômico futuro da Europa.

Em relação ao modelo 5, o fator de risco $C M A$, contrariando o valor médio negativo estimado por MQO, apresentou relação positiva nos percentis 0,05 e 0,50. Porém, observou-se a existência de significância estatística, ao nível de $5 \%$, no percentil inferior $(0,05)$, em que a magnitude do coeficiente foi de 2,6\%. Estes resultados contariam os observados por Ferreira e Gama (2020) e evidencia a robustez do fator de risco CMA dos mercados de ações emergentes.

Os resultados reportados na Tabela 2 evidenciam que, para o período em análise (1993 a 2019), os fatores de risco dos mercados de ações emergentes, de forma individual, ajudam a prever o crescimento econômico conjunto dos BRICS, em linha com o estudo de Ferreira e Gama (2020) para os mercados regionais da América do Norte, Ásia Pacifico e Europa.

A Tabela 3 apresenta as estimações do modelo de cinco variáveis preditoras, associado ao modelo de avaliação de ativos de cinco fatores, representado pela equação (Eq. 3).

Tabela 3. Estimações da regressão múltipla para o modelo $P I B_{t}=\alpha+\beta_{1} M K T_{t-1}+$ $\beta_{2} S M B_{t-1}+\beta_{3} H M L_{t-1}+\beta_{4} R M W_{t-1}+\beta_{5} C M A_{t-1}+\varepsilon_{t}$

\begin{tabular}{|c|c|c|c|c|c|c|}
\hline \multirow[b]{2}{*}{ Modelo } & \multirow[b]{2}{*}{ MQO } & \multicolumn{5}{|c|}{ Regressão quantílica } \\
\hline & & 0,05 & 0,25 & $\mathbf{0 , 5 0}$ & 0,75 & 0,95 \\
\hline \multicolumn{7}{|l|}{ BRICS } \\
\hline MKT & $\begin{array}{c}0,033 * * * \\
(0,008)\end{array}$ & $\begin{array}{c}0,050 * * * \\
(0,000)\end{array}$ & $\begin{array}{c}0,020 * * * \\
(0,001)\end{array}$ & $\begin{array}{c}0,027 * * * \\
(0,001)\end{array}$ & $\begin{array}{c}0,024 * * * \\
(0,000)\end{array}$ & $\begin{array}{c}0,042 * * * \\
(0,005)\end{array}$ \\
\hline SMB & $\begin{array}{c}-0,054 \\
(0,066) \\
\end{array}$ & $\begin{array}{c}0,054 * * * \\
(0,001)\end{array}$ & $\begin{array}{c}-0,015^{* *} \\
(0,006) \\
\end{array}$ & $\begin{array}{c}-0,078 * * * \\
(0,007) \\
\end{array}$ & $\begin{array}{c}-0,081 * * * \\
(0,000) \\
\end{array}$ & $\begin{array}{c}-0,150 * * * \\
(0,024) \\
\end{array}$ \\
\hline HML & $\begin{array}{c}0,007 \\
(0,034)\end{array}$ & $\begin{array}{c}0,081 * * * \\
(0,000)\end{array}$ & $\begin{array}{c}0,025 * * * \\
(0,004) \\
\end{array}$ & $\begin{array}{c}0,016 * * * * \\
(0,004) \\
\end{array}$ & $\begin{array}{c}0,018^{* * * *} \\
(0,000) \\
\end{array}$ & $\begin{array}{l}-0,014 \\
(0,016) \\
\end{array}$ \\
\hline RMW & $\begin{array}{l}0,032 \\
0,067\end{array}$ & $\begin{array}{c}0,202 * * * \\
(0,001)\end{array}$ & $\begin{array}{c}0,077 * * * * \\
(0,006)\end{array}$ & $\begin{array}{c}0,036 * * * * \\
(0,007) \\
\end{array}$ & $\begin{array}{c}-0,049 * * * \\
(0,000) \\
\end{array}$ & $\begin{array}{l}-0,138^{*} \\
(0,027)\end{array}$ \\
\hline CMA & $\begin{array}{c}-0,026 \\
(0,036) \\
\end{array}$ & $\begin{array}{c}0,029 * * * \\
(0,001)\end{array}$ & $\begin{array}{c}0,018 * * * \\
(0,004)\end{array}$ & $\begin{array}{c}-0,033 * * * \\
(0,005) \\
\end{array}$ & $\begin{array}{c}-0,049 * * * \\
(0,000) \\
\end{array}$ & $\begin{array}{l}-0,033^{*} \\
(0,019) \\
\end{array}$ \\
\hline \multicolumn{7}{|c|}{ África do Sul } \\
\hline MKT & $\begin{array}{c}0,030 * * \\
(0,008)\end{array}$ & $\begin{array}{c}0,037 * * * * \\
(0,003)\end{array}$ & $\begin{array}{c}0,027 * * * \\
(0,009)\end{array}$ & $\begin{array}{c}0,016 \\
(0,010)\end{array}$ & $\begin{array}{c}0,025 * * * \\
(0,012)\end{array}$ & $\begin{array}{c}0,041 * * * \\
(0,006)\end{array}$ \\
\hline SMB & $\begin{array}{c}0,027 \\
(0,064)\end{array}$ & $\begin{array}{c}0,097 * * * \\
(0,015) \\
\end{array}$ & $\begin{array}{c}0,100^{* *} \\
(0,040)\end{array}$ & $\begin{array}{l}0,082 * \\
(0,046)\end{array}$ & $\begin{array}{c}-0,113^{* *} \\
(0,056)\end{array}$ & $\begin{array}{c}-0,082 * * * \\
(0,029)\end{array}$ \\
\hline HML & $\begin{array}{c}0,032 \\
(0,032)\end{array}$ & $\begin{array}{c}0,063 * * * \\
(0,010) \\
\end{array}$ & $\begin{array}{c}0,075^{* *} \\
(0,027) \\
\end{array}$ & $\begin{array}{c}0,065^{* *} \\
(0,030)\end{array}$ & $\begin{array}{l}-0,002 \\
(0,037) \\
\end{array}$ & $\begin{array}{l}-0,015 \\
(0,019) \\
\end{array}$ \\
\hline RMW & $\begin{array}{c}0,074 \\
(0,066)\end{array}$ & $\begin{array}{c}0,170 * * * \\
(0,017) \\
\end{array}$ & $\begin{array}{c}0,157 * * * \\
(0,046) \\
\end{array}$ & $\begin{array}{l}0,132 * * \\
(0,052)\end{array}$ & $\begin{array}{l}-0,046 \\
(0,063)\end{array}$ & $\begin{array}{c}0,000 \\
(0,032)\end{array}$ \\
\hline CMA & $\begin{array}{c}0,040 \\
(0,038)\end{array}$ & $\begin{array}{c}0,081 * * * \\
(0,012)\end{array}$ & $\begin{array}{l}0,086^{* * *} \\
(0,032)\end{array}$ & $\begin{array}{l}0,073 * \\
(0,036) \\
\end{array}$ & $\begin{array}{l}-0,063 \\
(0,044) \\
\end{array}$ & $\begin{array}{l}-0,046^{*} \\
(0,023)\end{array}$ \\
\hline
\end{tabular}




\begin{tabular}{|c|c|c|c|c|c|c|}
\hline \multicolumn{7}{|l|}{ Brasil } \\
\hline MKT & $\begin{array}{c}0,049 * * * \\
(0,007)\end{array}$ & $\begin{array}{c}0,041 * * * \\
(0,006)\end{array}$ & $\begin{array}{c}0,043 \text { *** } \\
(0,007)\end{array}$ & $\begin{array}{c}0,040 * * * \\
(0,008)\end{array}$ & $\begin{array}{c}0,048 * * * \\
(0,000)\end{array}$ & $\begin{array}{c}0,027 * * * \\
(0,000)\end{array}$ \\
\hline SMB & $\begin{array}{c}0,021 \\
(0,053)\end{array}$ & $\begin{array}{c}-0,193 * * * \\
(0,026)\end{array}$ & $\begin{array}{c}0,110 \text { *** } \\
(0,034)\end{array}$ & $\begin{array}{c}0,058 \\
(0,035)\end{array}$ & $\begin{array}{c}0,017 * * * \\
(0,000)\end{array}$ & $\begin{array}{c}0,130 * * * \\
(0,002)\end{array}$ \\
\hline HML & $\begin{array}{c}0,052 \\
(0,036) \\
\end{array}$ & $\begin{array}{c}0,048 * * * \\
(0,017)\end{array}$ & $\begin{array}{c}0,085^{* * *} * \\
(0,023)\end{array}$ & $\begin{array}{c}0,024 \\
(0,023) \\
\end{array}$ & $\begin{array}{c}0,027 * * * \\
(0,000)\end{array}$ & $\begin{array}{c}-0,009 * * * \\
(0,001)\end{array}$ \\
\hline RMW & $\begin{array}{c}0,063 \\
(0,050)\end{array}$ & $\begin{array}{c}-0,165 * * * \\
(0,029)\end{array}$ & $\begin{array}{c}0,158 * * \\
(0,039)\end{array}$ & $\begin{array}{l}0,078 * \\
(0,039)\end{array}$ & $\begin{array}{c}0,066 * * * \\
(0,000)\end{array}$ & $\begin{array}{c}-0,123 * * * \\
(0,002)\end{array}$ \\
\hline CMA & $\begin{array}{c}0,032 \\
(0,030)\end{array}$ & $\begin{array}{c}0,117 * * * \\
(0,020)\end{array}$ & $\begin{array}{c}0,065 * * * \\
(0,027)\end{array}$ & $\begin{array}{c}0,018 \\
(0,028)\end{array}$ & $\begin{array}{c}-0,015 * * * \\
(0,000)\end{array}$ & $\begin{array}{c}0,084 * * * \\
(0,001)\end{array}$ \\
\hline \multicolumn{7}{|l|}{ China } \\
\hline MKT & $\begin{array}{c}0,011 \\
(0,007)\end{array}$ & $\begin{array}{c}0,022 * * * \\
(0,003)\end{array}$ & $\begin{array}{c}0,017 * * * \\
(0,000)\end{array}$ & $\begin{array}{c}0,011 \\
(0,008)\end{array}$ & $\begin{array}{c}0,015^{* * *} * \\
(0,002)\end{array}$ & $\begin{array}{c}0,019 * * * \\
(0,000)\end{array}$ \\
\hline SMB & $\begin{array}{c}0,027 \\
(0,076) \\
\end{array}$ & $\begin{array}{c}0,145^{* * *} \\
(0,016)\end{array}$ & $\begin{array}{c}0,109 * * * \\
(0,000)\end{array}$ & $\begin{array}{c}0,124 * * * \\
(0,038)\end{array}$ & $\begin{array}{c}-0,092 * * * \\
(0,010)\end{array}$ & $\begin{array}{c}-0,195 * * * \\
(0,001)\end{array}$ \\
\hline HML & $\begin{array}{c}0,031 \\
(0,036) \\
\end{array}$ & $\begin{array}{c}0,105^{* * *} \\
(0,010)\end{array}$ & $\begin{array}{c}0,084 * * * \\
(0,000)\end{array}$ & $\begin{array}{c}0,083 * * * \\
(0,025)\end{array}$ & $\begin{array}{c}-0,023 * * * \\
(0,006) \\
\end{array}$ & $\begin{array}{c}-0,052 * * * \\
(0,001)\end{array}$ \\
\hline RMW & $\begin{array}{l}-0,015 \\
(0,082)\end{array}$ & $\begin{array}{c}0,141 \text { *** } \\
(0,018)\end{array}$ & $\begin{array}{c}0,093 * * * \\
(0,000)\end{array}$ & $\begin{array}{c}0,106 * * \\
(0,043)\end{array}$ & $\begin{array}{c}-0,116^{* * *} \\
(0,011) \\
\end{array}$ & $\begin{array}{c}-0,203 * * * \\
(0,001) \\
\end{array}$ \\
\hline CMA & $\begin{array}{c}0,009 \\
(0,039)\end{array}$ & $\begin{array}{c}0,059 * * * \\
(0,012)\end{array}$ & $\begin{array}{c}0,039 * * * \\
(0,000)\end{array}$ & $\begin{array}{c}0,046 \\
(0,030)\end{array}$ & $\begin{array}{c}-0,063 * * * \\
(0,008)\end{array}$ & $\begin{array}{c}-0,124 * * * \\
(0,001)\end{array}$ \\
\hline \multicolumn{7}{|l|}{ Índia } \\
\hline MKT & $\begin{array}{c}-0,008 \\
(0,011) \\
\end{array}$ & $\begin{array}{l}-0,010 \\
(0,011) \\
\end{array}$ & $\begin{array}{l}-0,010 \\
(0,021)\end{array}$ & $\begin{array}{c}-0,014 \\
(0,010) \\
\end{array}$ & $\begin{array}{c}-0,018 * * * \\
(0,001)\end{array}$ & $\begin{array}{c}-0,026 * * * \\
(0,002) \\
\end{array}$ \\
\hline SMB & $\begin{array}{l}-0,062 \\
(0,049) \\
\end{array}$ & $\begin{array}{l}-0,019 \\
(0,052) \\
\end{array}$ & $\begin{array}{l}-0,079 \\
(0,097) \\
\end{array}$ & $\begin{array}{c}-0,069 \\
(0,046) \\
\end{array}$ & $\begin{array}{c}-0,023 * * * \\
(0,005) \\
\end{array}$ & $\begin{array}{c}-0,041 * * * \\
(0,011) \\
\end{array}$ \\
\hline HML & $\begin{array}{l}-0,022 \\
(0,032)\end{array}$ & $\begin{array}{c}0,021 \\
(0,034)\end{array}$ & $\begin{array}{l}-0,053 \\
(0,063)\end{array}$ & $\begin{array}{c}-0,048 \\
(0,031)\end{array}$ & $\begin{array}{c}0,002 \\
(0,003)\end{array}$ & $\begin{array}{c}0,040 * * * \\
(0,007)\end{array}$ \\
\hline RMW & $\begin{array}{c}-0,103 * \\
(0,056)\end{array}$ & $\begin{array}{c}-0,077 \\
(0,059) \\
\end{array}$ & $\begin{array}{l}-0,148 \\
(0,109) \\
\end{array}$ & $\begin{array}{c}-0,121 * * \\
(0,052)\end{array}$ & $\begin{array}{c}-0,035 * * * \\
(0,005)\end{array}$ & $\begin{array}{c}-0,022 * \\
(0,013) \\
\end{array}$ \\
\hline CMA & $\begin{array}{c}-0,093 * * \\
(0,039)\end{array}$ & $\begin{array}{c}-0,178 \\
(0,041)\end{array}$ & $\begin{array}{l}-0,066 \\
(0,076)\end{array}$ & $\begin{array}{l}-0,106 \\
(0,037)\end{array}$ & $\begin{array}{l}-0,103 \\
(0,004)\end{array}$ & $\begin{array}{l}-0,094 \\
(0,009)\end{array}$ \\
\hline \multicolumn{7}{|l|}{ Rússia } \\
\hline MKT & $\begin{array}{c}0,089 * * \\
(0,032)\end{array}$ & $\begin{array}{c}0,107 * * * \\
0,005\end{array}$ & $\begin{array}{c}0,121 * * * \\
0,040\end{array}$ & $\begin{array}{c}0,068 * * * \\
0,007\end{array}$ & $\begin{array}{l}0,010 \\
0,008 \\
\end{array}$ & $\begin{array}{c}-0,005^{* *} \\
0,002\end{array}$ \\
\hline SMB & $\begin{array}{c}-0,111 \\
(0,146)\end{array}$ & $\begin{array}{c}0,261 * * * \\
0,022\end{array}$ & $\begin{array}{l}0,027 \\
0,187\end{array}$ & $\begin{array}{c}-0,159 * * * \\
0,034\end{array}$ & $\begin{array}{c}-0,017 \\
0,038 \\
\end{array}$ & $\begin{array}{c}-0,011 \\
0,011 \\
\end{array}$ \\
\hline HML & $\begin{array}{c}0,048 \\
(0,096) \\
\end{array}$ & $\begin{array}{c}0,406^{* * *} \\
0,015 \\
\end{array}$ & $\begin{array}{l}0,137 \\
0,123 \\
\end{array}$ & $\begin{array}{c}0,049 * * \\
0,022\end{array}$ & $\begin{array}{c}0,077 * * * \\
0,025\end{array}$ & $\begin{array}{c}0,021 * * * \\
0,007\end{array}$ \\
\hline RMW & $\begin{array}{l}0,298 * \\
(0,164)\end{array}$ & $\begin{array}{c}0,869 * * * \\
0,025 \\
\end{array}$ & $\begin{array}{c}0,554 * * \\
0,210\end{array}$ & $\begin{array}{c}0,308 * * * \\
0,038 \\
\end{array}$ & $\begin{array}{c}-0,144 * * * \\
0,043 \\
\end{array}$ & $\begin{array}{c}-0,232 * * * \\
0,012 \\
\end{array}$ \\
\hline CMA & $\begin{array}{c}0,028 \\
(0,115) \\
\end{array}$ & $\begin{array}{c}0,128 * * * \\
0,018\end{array}$ & $\begin{array}{l}0,190 \\
0,147\end{array}$ & $\begin{array}{c}-0,029 \\
0,027\end{array}$ & $\begin{array}{c}0,161 * * * \\
0,030\end{array}$ & $\begin{array}{c}0,137 * * * \\
0,009\end{array}$ \\
\hline
\end{tabular}

Nota: MQO, mínimos quadrados ordinários; ***, **, *, P < 1\%, 5\% e 10\%. Erros-padrão entre parênteses.

Fonte: Autores, 2021.

Por meio da análise da Tabela 3, constata-se que, em relação ao PIB conjunto dos BRICS, a centralidade do fator de risco $M K T$ evidenciou-se tanto pela relação positiva como pela significância estatística, ao nível de 5\%, em todos os percentis em análise. Esta análise se estende para o desempenho econômico futuro do Brasil. Com efeito, para o PIB conjunto dos 
BRICS, a magnitude dos coeficientes variou entre $2 \%$ (percentil 0,25 ) a $5 \%$ (percentil 0,05 ), em comparação ao valor médio de 3,3\% (estatisticamente significante ao nível de 5\%) obtido por meio da estimação por MQO. Para o Brasil, os coeficientes estimados apresentaram magnitude da ordem de $2,7 \%$ (percentil 0,95 ) a $4,8 \%$ (percentil 0,75 ), contra um valor médio de 4,9\% e estatisticamente significante, ao nível de 5\%, obtido pela estimação por MQO.

Em relação ao desempenho econômico dos restantes países em análise, a exceção da Índia, o fator de risco $M K T$ apresentou uma relação positiva e estatisticamente significante, ao nível de $5 \%$, para todos os países, bem como entre três a quatro percentis para Rússia, África do Sul e China, com magnitude dos coeficientes da ordem de 1,7\% (China - percentil 0,25) a 12,1\% (Rússia - percentil 0,25), em comparação ao valor médio de 8,9\% (Rússia) e estatisticamente significante, ao nível de $5 \%$, obtido por meio da estimação por MQO. Estes resultados estão em linha com os obtidos por Ferreira e Gama (2020) para o desempenho econômico das regiões da América do Norte, Ásia Pacífico e Europa, como Alemanha, Canadá, Estados Unidos, França, Hong Kong e Singapura.

O fator $S M B$ apresentou coeficientes positivos e estatisticamente significantes, ao nível de 5\%, em um percentil para o PIB conjunto dos BRICS $(5,4 \%$ - percentil 0,05) e para a Rússia (26,1\% - percentil 0,05). Para os países restantes, $S M B$ apresentou uma relação positiva e estatisticamente significante, ao nível de 5\%, em três dos cinco percentis, para África do Sul, Brasil e China, com magnitude dos coeficientes da ordem de 1,7\% (Brasil - percentil 0,75) a 14,5\% (China - percentil 0,05), em comparação ao valor médio (sem significância estatística ao nível de 10\%) obtido por meio de MQO. Ferreira e Gama (2020) constataram desempenho positivo e significante de $S M B$ para o crescimento econômico da região da Ásia Pacífico, assim como para Alemanha, Hong Kong e Singapura.

Para o fator de risco $H M L$, verificou-se a relação positiva e estatisticamente significante, ao nível de 5\%, em quatro dos cinco percentis em análise para o desempenho econômico conjunto dos BRICS e da Rússia, com magnitude dos coeficientes variando entre 1,6\% (BRICS - percentil 0,50) e 40,6\% (Rússia - percentil 0,05), em comparação a valores médios e sem significância estatística (ao nível de 10\%) estimados por MQO.

Em relação ao desempenho dos restantes países, contrariando as estimações por MQO, da qual não apresentaram significância estatística, $H M L$ apresentou coeficientes positivos e estatisticamente significantes, ao nível de 5\%, entre um a três percentis para Índia, África do Sul, Brasil e China, com magnitude variando entre 2,7\% (Brasil - percentil 0,75) e 10,5\% (China - percentil 0,05). Estes resultados estão em linha com os de Ferreira e Gama (2020) para as regiões da América do Norte, Ásia Pacífico e Europa, e para o PIB doméstico de Alemanha, Canadá, França, Hong Kong e Singapura.

O fator de risco $R M W$ apresentou coeficientes positivos e estatisticamente significantes, ao nível de 5\%, em três dos cinco percentis para o PIB conjunto dos BRICS, África do Sul, Brasil, China e Rússia, com magnitude variando entre 3,6\% (BRICS - percentil 0,50) e 86,9\% (Rússia - percentil 0,05), em comparação ao valor médio de 29,8\% (estatisticamente significante ao nível de 10\%) obtido por meio da estimação por MQO. Estes resultados estão em linha com os de Ferreira e Gama (2020) para as regiões da Ásia Pacífico e Europa, bem como para Alemanha, França, Hong Kong e Singapura.

Por fim, para o fator de risco $C M A$, a exceção da Índia, verificou-se a existência de um desempenho positivo e estatisticamente significante, ao nível de 5\%, para o PIB conjunto dos BRICS e para todos os países, entre dois a três percentis. A magnitude dos coeficientes variou entre 1,8\% (BRICS - percentil 0,25) e 12,8\% (Rússia - percentil 0,05), em comparação aos valores médios (sem significância estatística ao nível de 10\%) obtidos por meio de MQO. Ferreira e Gama (2020) observaram relação positiva e estatisticamente significante entre o fator de CMA de mercados de ações desenvolvidos de natureza regional e o desempenho econômico futuro para a região da Ásia Pacifico, bem como para Alemanha, Hong Kong e Singapura. 


\section{CONCLUSÃO}

O objetivo deste estudo foi investigar se os fatores de risco de mercados de ações emergentes do modelo de avaliação de ativos de cinco fatores proposto por Fama e French (2015) captam informação que ajudam a prever o crescimento econômico do conjunto de países dos BRICS, bem como de cada um dos cinco países que compõem a sigla. Utilizando dados anuais dos retornos dos fatores de risco e da taxa de crescimento do PIB do período de janeiro de 1993 a dezembro de 2019, aplicou-se a técnica de regressão quantílica para os percentis 0,05; 0,$25 ; 0,50 ; 0,75$; e 0,95 , que permitiu verificar o comportamento da distribuição assimétrica condicional da taxa de crescimento do PIB produzido pelos retornos dos fatores de risco.

O método adotado teve como base os estudos empíricos de Liew e Vassalou (2000), Neves e Leal (2003), Hanhardt e Ansotegui Olcoz (2008), que analisaram a relação entre o crescimento econômico futuro e o modelo de três fatores de Fama e French (1993) no contexto de mercados de ações domésticos. Os resultados empíricos obtidos pela regressão quantílica reportaram informações não captadas pela média condicional gerada por estimações MQO. Este fato permite concluir, para o período em análise e a exceção da Índia, que os fatores de risco de mercados de ações emergentes do modelo de avaliação de ativos proposto por Fama e French (2015) e desenvolvidos para estimar o custo de capital e carteiras de investimento podem servir com proxies de indicadores econômicos. O fator de risco $M K T$, em linha com os achados de Ferreira e Gama (2020), de forma individual e em conjunto com outros fatores de risco, oferece estimações significantes que reforçam a sua centralidade na captação dos fatores macroeconômicos, tal como observado por Levy e Roll (2010).

Para além do estudo de Ferreira e Gama (2020), que se limitou analisar a relação entre o crescimento econômico futuro de países de mercados de ações desenvolvidos e os fatores de risco do modelo de avaliação de ativos de cinco fatores, não se encontraram estudos que caminharam nesta direção. Esta pesquisa, neste sentido, vem complementar os estudos já publicados que documentaram a importância dos fatores de risco $M K T, S M B$ e $H M L$ como ferramentas que auxiliam na previsão do crescimento econômico, contribuindo para a literatura sobre a validação dos modelos de avaliação de ativos de fatores de risco comum e lançando luz sobre a integração dos mercados de ações emergentes.

Os retornos dos fatores de risco de mercados emergentes são formados por ações de 26 países. Ao se estudar uma amostra de cinco países entre 1993 e 2019, o estudo apresenta limitações, o que faz com que seja sugerida, para futuras pesquisas, a ampliação da análise para um horizonte temporal mais longo e para os demais países de mercados de ações emergentes.

\section{REFERÊNCIAS}

Aylard, A., \& Glen, J. (2000). Some international evidence on stock prices as leading indicators of economic activity. Applied Financial Economics, 10(1), 1-14.

Brooks, R., \& Del Negro, M. (2005). Country versus region effects in international stock returns. Journal of Portfolio Management, 31(4), 67-72.

Buchinsky, M. (1998). Recent advances in quantile regression models: a practical guideline for empirical research. Journal of Human Resources, 88-126.

Capaul, C., Rowley, I., \& Sharpe, W. F. (1993). International value and growth stock returns. Financial Analysts Journal, 49(1), 27-36.

Chauvet, M. (1999). Stock market fluctuations and the business cycle. Journal of Economic and Social Measurement, 25(3-4), 235-257. 
Chen, N., Roll, R., \& Roos, S. (1986). Economics forces and stock market. Journal of Business, 59(3), 383-403.

Erdinç, Y. (2017). Comparison of CAPM, three factor Fama-French model and Five-Factor Fama-French model for the Turkish stock market. Financial Management from an Emerging Market Perspective, 69-92.

Fama, E. F. (1970). Efficient capital markets: A review of theory and empirical work. Journal of Finance, 25(2), 383-417.

Fama, E. F. (1981). Stock returns, real activity, inflation and money. American Economic Review, 71(4), 545-565.

Fama, E. F. (1990). Stock returns, expected returns, and real activity. Journal of Finance, 45(4), 1089-1108.

Fama, E. F., \& French, K. (1998). Value versus growth: The international evidence. Journal of Finance, 53(6), 1975-1999.

Fama, E. F., \& French, K. (2015). A five-factor asset pricing model. Journal of Financial Economics, 116(1), 1-22.

Fama, E. F., \& French, K. R. (2012). Size, value, and momentum in international stock returns. Journal of Financial Economics, 105(3), 457-472.

Fama, E. F., \& French, R. (2017). International tests of a five-factor asset pricing model. Journal of Financial Economics, 123(3), 441-463.

Fama, F., \& French, K. (1993). Common risk factors in the returns of stock and bonds. Journal of Financial Economics, 33(1), 3-56.

Fávero, L., \& Belfiore, P. (2017). Manual de análise de dados. Estatística e modelagem multivariada com Excel, SPSS e Stata. Rio de Janeiro: Elsevier

Ferreira, J. C. J., \& Gama, A. P. M. (2020). The relationship between the factors of risk in asset evaluation models and future economic growth: evidence from three regional markets. Journal of Spatial and Organizational Dynamics, 8(4), 300-319.

Fischer, S., \& Merton, R. C. (1984). Macroeconomics and finance: The role of the stock market. Carnegie-Rochester Conference Series on Public Policy, 21(1), 57-108.

Guo, B., Zhang, W., Zhang, Y., \& Zhang, H. (2017). The five-factor asset pricing model tests for the Chinese stock market. Pacific-Basin Finance Journal, 43, 84-106.

Hanhardt, A., \& Ansotegui Olcoz, C. (2008). Do the Fama and French factors proxy for state variables that predict macroeconomic growth in the eurozone? Disponível em SSRN 1098225 .

Jegadeesh, N., \& Titman, S. (1993). Returns to buying winners and selling losers: implications for stock market efficiency. Journal of Finance, 48(1), 65-91.

Jensen, G. R., Johnson, R. R., \& Mercer, J. M. (1997). New evidence on size and price-to-book effects in stock returns. Financial Analysts Journal, 53(6), 34-42.

Karaomer, Y. (2017). Fama-French five factor model: evidence from Turkey. International Journal of Economics and Financial Issues, 7(6), 130.

Kaul, G. (1987). Stock returns and inflation: The role of the monetary sector. Journal of Financial Economics, 18(2), 253-276. 
Koenker, R., \& Basset Jr., G. (1978). Regression quantiles. Econometrica: Journal of the Econometric Society, 46(1) 33-50.

Leite, A. L., Klotzle, M. C., Pinto, A. C. F., \& Da Silva, A. F. (2018). Size, value, profitability, and investment: Evidence from emerging markets. Emerging Markets Review, 36, 45-59.

Levy, M. \& Roll, R. (2010). The market portfolio may be mean/variance efficient after all: the market portfolio. The Review of Financial Studies, 23(6), 2464-2491.

Liew, J., \& Vassalou, M. (2000). Can book-to-market, size and momentum be risk factors that predict economic growth?. Journal of Financial Economics, 57(2), 221-245.

Lin, Q. (2017). Noisy prices and the Fama-French five-factor asset pricing model in China. Emerging Markets Review, 31, 141-163.

Lintner, J. (1965). The valuation of risk assets and the selection of risky investments in stock portfolios and capital budgets. Review of Economics and Statistics, 47(1), 13-37.

Liu, B., \& Di Iorio, A. (2013). Do the asset pricing factors predict future economy growth? An Australian study. Proceedings of the European Financial Management Association Conference, Reading, UK, 22.

Markowitz, H. (1952). Portfolio selection. Journal of Finance, 7(1), 77-91.

Mauro, P. (2003) Stock returns and output growth in emerging and advanced economies. Journal of Development Economic, 71(1) 129-153.

Merton, R. C. (1973). An intertemporal capital asset pricing model. Econometrica: Journal of the Econometric Society, 41(5), 867-887.

Moerman, G. A. (2005). How domestic is the Fama and French three-factor model? An application to the Euro area. Research Paper ERS; ERS-2005-035-F\&A, Erasmus Research Institute of Management.

Morck, R., Shleifer, A., Vishny, R. W., Shapiro, M., \& Poterba, J. M. (1990). The stock market and investment: is the market a sideshow?. Brookings Papers on Economic Activity, $1990(2), 157-215$.

Neves, M., \& Leal, R. (2003). Existe relação entre o crescimento do PIB Brasileiro e os efeitos tamanho, valor e momento? Anais do Encontro Nacional da Associação Nacional de PósGraduação e Pesquisa em Administração, Atibaia, SP, 17.

Newey, W. K., \& West, K. D. (1987) A simple, positive semi-definite, heteroskedasticity and autocorrelation consistent covariance matrix. Econometrica, 55(3), 703-708.

Ozkan, N. (2018). Fama-French five factor model and the necessity Of value factor: Evidence from Istanbul stock exchange. Press Academia Procedia, 8(1), 14-17.

Panopoulou, E. (2009). Financial variables and euro area growth: a non-parametric causality analysis. Economic Modelling, 26(6), 1414-1419.

Schwert, G. W. (1989). Business cycles, financial crises, and stock volatility. CarnegieRochester Conference series on public policy, 31, 83-125.

Schwert, G. W. (1990). Stock returns and real activity: A century of evidence. Journal of Finance, 45(4), 1237-1257.

Sharpe, F. (1964). Capital asset prices: A theory of market equilibrium under conditions of risk. The Journal of Finance, 19(3), 425-442. 
Singh, S., \& Yadav, S. S. (2015). Indian stock market and the asset pricing models. Procedia Economics and Finance, 30, 294-304.

White, H. (1980). A heteroskedasticity-consistent covariance matrix estimator and a direct test for heteroskedasticity. Econometrica: Journal of the Econometric Society, 48, 817-838.

Zaremba, A., \& Czapkiewicz, A. (2017). Digesting anomalies in emerging European markets: A comparison of factor pricing models. Emerging Markets Review, 31, 1-15.

Zarnowitz, V. (1992). What is a business cycle?. In The business cycle: Theories and evidence, Springer Dordrecht, 3-83.

\section{APÊNDICE}

Para fins de comparação das magnitudes e dos sinais dos parâmetros das variáveis preditoras, a Tabela 4 apresenta os resultados dos modelos de regressão quantílica em painel com efeitos fixos, para diferentes percentis.

Tabela 4. Estimações de modelos de regressão em painel quantílico com efeitos fixos, para diferentes percentis $P I B_{i t}=\alpha_{i}+\beta_{1}$ Fator $_{i t-1}+\beta_{k}$ Dummies $_{i}$ em que Fator denota $M K T, S M B, H M L, R M W, C M A$, e Dummies os países dos BRICS para a estimação com efeitos fixos (parâmetros das variáveis dummy omitidos)

\begin{tabular}{|c|c|c|c|c|c|c|}
\hline & \multirow{2}{*}{$\begin{array}{l}\text { Variável } \\
\text { preditora }\end{array}$} & \multicolumn{5}{|c|}{ Painel quantílica com efeitos fixos } \\
\hline & & $\mathbf{0 , 0 5}$ & 0,25 & $\mathbf{0 , 5 0}$ & 0,75 & $\mathbf{0 , 9 5}$ \\
\hline 1 & MKT & $\begin{array}{c}0,022 \\
(0,014)\end{array}$ & $\begin{array}{c}0,029 * * * \\
(0,010)\end{array}$ & $\begin{array}{c}0,023 * * * \\
(0,007)\end{array}$ & $\begin{array}{c}0,019 * * \\
(0,008)\end{array}$ & $\begin{array}{c}0,033 * * * \\
(0,005)\end{array}$ \\
\hline 2 & SMB & $\begin{array}{c}0,042 \\
(0,066)\end{array}$ & $\begin{array}{c}0,048 \\
(0,047)\end{array}$ & $\begin{array}{c}0,046 \\
(0,033)\end{array}$ & $\begin{array}{c}-0,070 * \\
(0,037)\end{array}$ & $\begin{array}{c}-0,102 * * * \\
(0,024)\end{array}$ \\
\hline 3 & HML & $\begin{array}{c}0,134 * * * \\
(0,045)\end{array}$ & $\begin{array}{l}0,061 * \\
(0,032)\end{array}$ & $\begin{array}{c}0,051 * * \\
(0,022)\end{array}$ & $\begin{array}{c}-0,004 \\
(0,025)\end{array}$ & $\begin{array}{c}-0,043^{* *} \\
(0,017)\end{array}$ \\
\hline 4 & RMW & $\begin{array}{c}0,329 * * * \\
(0,079)\end{array}$ & $\begin{array}{c}0,118 * * \\
(0,057)\end{array}$ & $\begin{array}{l}0,072 * \\
(0,040)\end{array}$ & $\begin{array}{l}-0,079 * \\
(0,044)\end{array}$ & $\begin{array}{c}-0,149 * * * \\
(0,029)\end{array}$ \\
\hline 5 & CMA & $\begin{array}{c}0,073 \\
(0,050) \\
\end{array}$ & $\begin{array}{c}0,025 \\
(0,036) \\
\end{array}$ & $\begin{array}{l}0,042 * \\
(0,025)\end{array}$ & $\begin{array}{c}-0,041 \\
(0,028) \\
\end{array}$ & $\begin{array}{c}-0,046^{* *} \\
(0,019)\end{array}$ \\
\hline
\end{tabular}

Nota: $* * *, * *, *, \mathrm{P}<1 \%, 5 \%$ e $10 \%$. Erros-padrão entre parênteses.

Por meio da Figura 2 é possível comparar a qualidade do ajuste do modelo de regressão quantílica (apresentado na Tabela 2) com a qualidade do ajuste do modelo de regressão em painel quantílico com efeitos fixos, para um percentil de 50\% apenas para fins ilustrativos (variável dependente real X fitted values). Por meio da referida figura, é possível verificar que não existem substanciais diferenças entre as qualidades das duas estimações. 


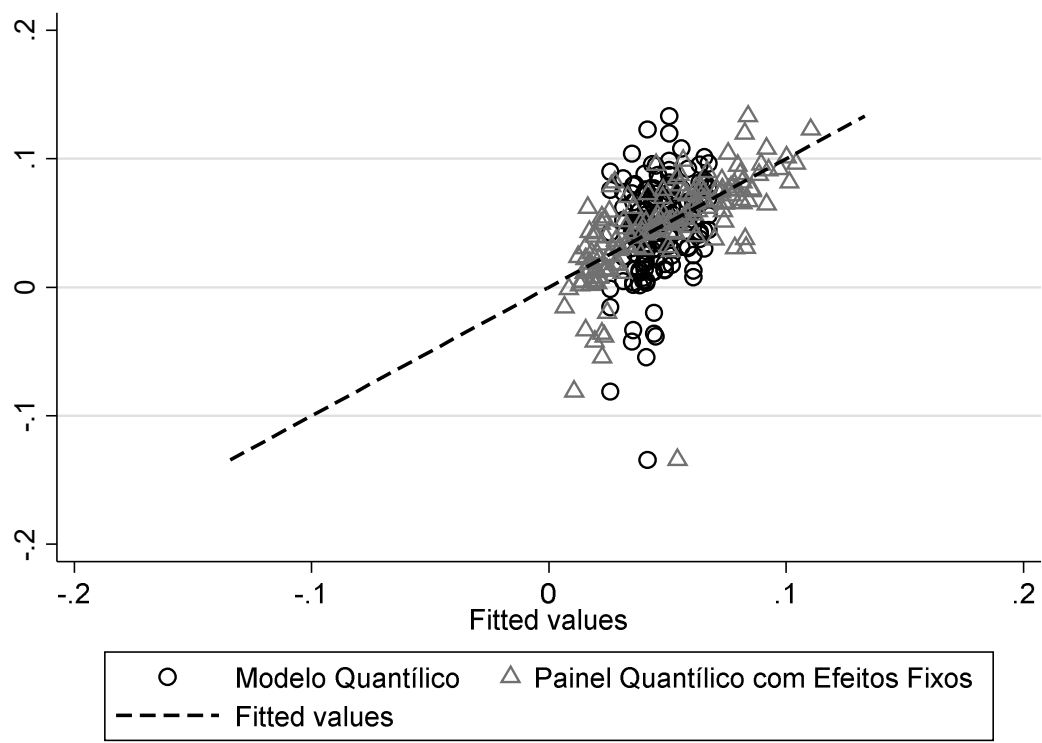

Figura 2. Comparação entre as qualidades dos ajustes dos modelos de regressão quantílica e de painel quantílico com efeitos fixos. Fonte: Autores, 2021. 